\title{
Standardized Solution for Management Controller for MTCA.4
}

\author{
D. Makowski, Member, IEEE, M. Fenner, F. Ludwig, U. Mavrič, A. Mielczarek, \\ A. Napieralski, Senior Member, IEEE, P. Perek, H. Schlarb
}

\begin{abstract}
The Micro Telecommunications Computing Architecture (MTCA) standard is a modern platform that is gaining popularity in the area of High Energy Physics (HEP) experiments. The standard provides extensive management, monitoring and diagnostics functionalities. The hardware control and monitoring is based on the Intelligent Platform Management Interface (IPMI), that was initially developed for supervision of complex computers operation. The original IPMI specification was extended to support functions required by the MTCA specification. The Module Management Controller (MMC) is required on each Advanced Mezzanine Card (AMC) installed in MTCA chassis. The Rear Transition Modules (RTMs) have to be equipped with RTM Management Controllers (RMCs) which is required by the MTCA.4 subsidiary specification. The commercially available implementations of MMC and RMC are expensive and do not provide the complete functionality that is required by specific HEP applications. Therefore, many research centers and commercial companies work on their own implementation of AMC and RTM controllers. The available implementations suffer because of lack of common approach and interoperability problems.

Since both Lodz University of Technology (TUL) and Deutsches Elektronen-Synchrotron (DESY) have long-term experience in developing ATCA and MTCA hardware, the authors decided to develop a unified solution of management controller fully compliant with AMC and MTCA.4 standards. The MMC v1.00 solution is dedicated for management of AMC and RTM modules. The MMC v1.00 is based on Atmel ATxmega MCUs and can be fully customized by the user or used as a drop-in-module without any modifications. The paper discusses the functionality of the MMC $v 1.00$ solution. The implementation was verified with developed evaluation kits for AMC and RTM cards.
\end{abstract}

Index Terms-Module Management Controller, Intelligent Platform Management Interface, Advanced Mezzanine Card, Rear Transition Module, Micro Telecommunications Computing Architecture, MTCA.4, High-Energy Physics

\section{INTRODUCTION}

$\mathbf{T}$ HE Micro Telecommunications Computing Architecture (MTCA) is a compact, modular, cost-effective standard dedicated for demanding applications in telecommunications and computing systems [1]. The main advantage of this architecture is high-level reliability, availability and maintainability. It is achieved by the use of redundant components and the advanced management based on the Intelligent Platform Management Interface (IPMI) [2], [3].

Manuscript received June 6, 2014; revised January 5, 2015.

D. Makowski, A. Mielczarek, A. Napieralski, P. Perek are with the Lodz University of Technology (TUL), Department of Microelectronics and Computer Science (DMCS), Lodz, Poland (e-mail: dmakow@dmcs.p.lodz.pl)

M. Fenner, F. Ludwig, U. Mavrič, H. Schlarb are with the Deutsches Elektronen-Synchrotron (DESY), Hamburg, Germany
Thanks to all the aforementioned features, application of the MTCA standard is gaining popularity in large-scale research facilities of High Energy Physics [4], [5]. In order to fulfil the requirements of such a systems, the MTCA.4 subsidiary specification has been developed. Beside the new features related to precise synchronization of system components, the MTCA.4 also introduces a new type of module, the Rear Transition Module (RTM). The RTM is connected to the front Advanced Mezzanine Card (AMC) [6] and is fully managed by this board. The AMC is also responsible for representation of the RTM for the system management. It causes the management in the MTCA.4 chassis to be more complicated as the Module Management Controllers (MMCs) of the front boards must implement additional logic dedicated for handling of the RTM [7]. Increasing complexity and demands for extended functionality cause many difficulties in providing correct implementation of the MMC firmware and can hence lead to serious stability issues, compromising the system reliability and availability [8]. For this reason the authors decided to design an unified solution for management controllers for AMC and RTM modules to facilitate the design of MTCA.4-compliant boards and to avoid problems related to the MMC development.

\section{A. Intelligent Platform Management Interface Standard}

The Intelligent Platform Management Interface (IPMI) was developed for out-of-band management and monitoring of complex computer systems [9]. The idea of IPMI is to provide the diagnostics of main components (e.g. Power Supply or Fan Tray Unit) that allows predicting and preventing failures. The IPMI operates independently of the host Operating System and allows remote administration of the computer even in case the main CPU is not operational.

A typical IPMI system is composed of the main Baseboard Management Controller (BMC) and satellite management controllers distributed among different subsystems. All devices communicate using the IPMI bus and message-based protocol. Each IPMI subsystem consists of Field Replaceable Units (FRUs) supervised by a microcontroller, that holds the inventory data (like device type, ID and manufacturer) and a Sensor Data Record (SDR) repository that describes the available sensors, e.g. temperature, voltage and fan speed.

\section{B. Management Controller in MTCA System}

The hardware platform management of the MTCA system is based on the IPMI specification. The system is composed of 


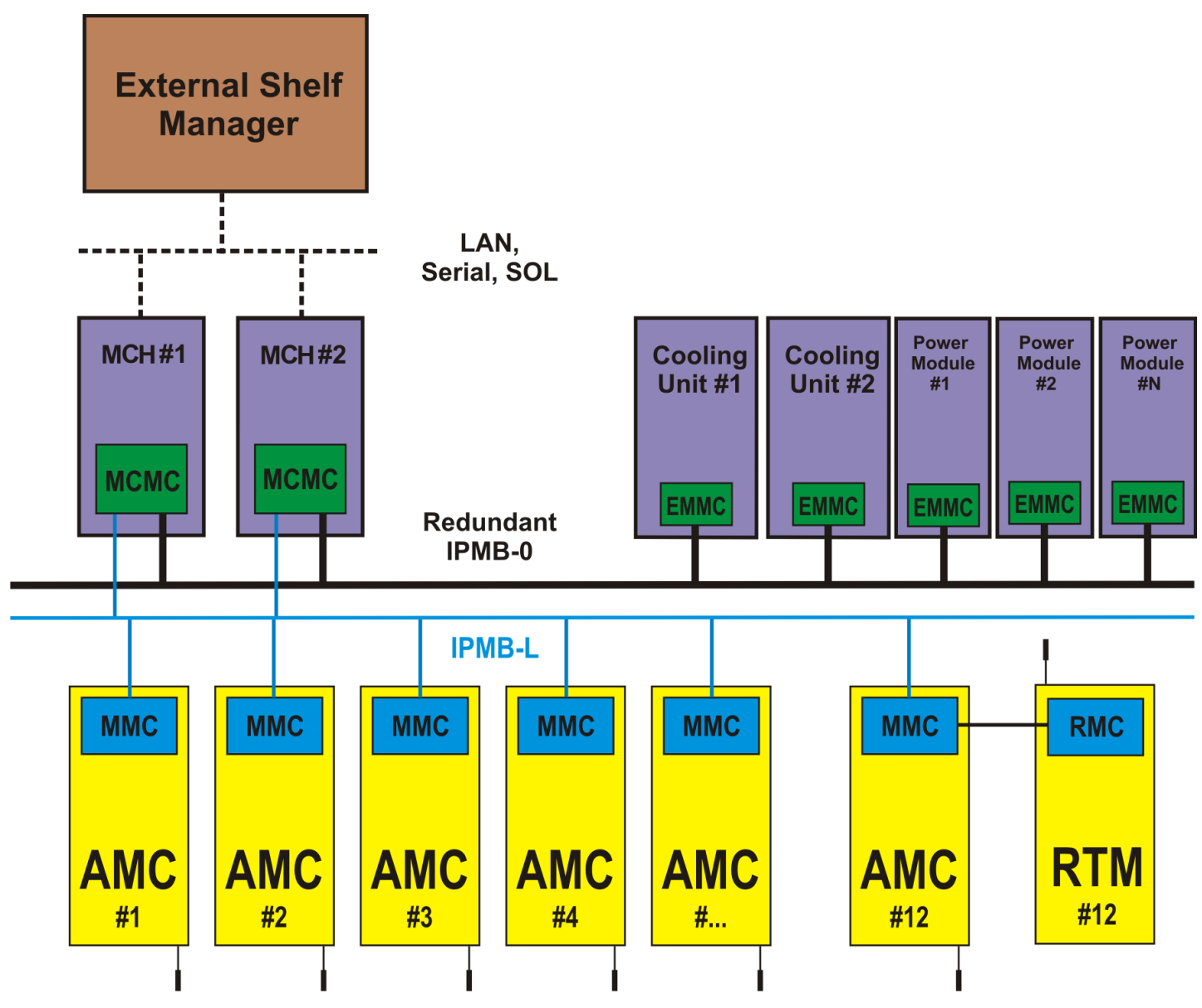

Fig. 1. A block diagram of the MTCA hardware management

one or several MCH (MTCA Carrier Hub) units that provide the Carrier Manager (CM) functionality, a MTCA Carrier Management Controller (MCMC) and distributed Module Management Controllers (MMCs) [1], [6]. Power modules and Cooling units are managed by EMMC (Enhanced Module Management Controller) that is connected via redundant IPMB-0 bus, whereas AMC modules are controlled by MMCs connected via IPMB-L bus. The block diagram of the MTCA system is presented in Figure 1.

The IPMI commands have been extended and adjusted for the requirements of the MTCA to support its hardwarespecific functionality. More detailed description of the MTCA management can be found in [1], [6], [10].

\section{MTCA.4 - EXTENDED FunCTIONALITY}

The MTCA.4 specification introduces the concept of an RTM that extends the functionality of a standard AMC card and allows connecting IO signals from the rear of the chassis [11]. An RTM module is connected with the AMC card via a Zone 3 connector as presented in Figure 2. The Zone 3 connector provides power supply, management and user signals.

The MTCA.4 requires extending the functionality of the MMC and the MCMC, that is a part of Shelf Manager, to control the RTM. In addition a simple IPMI circuitry is

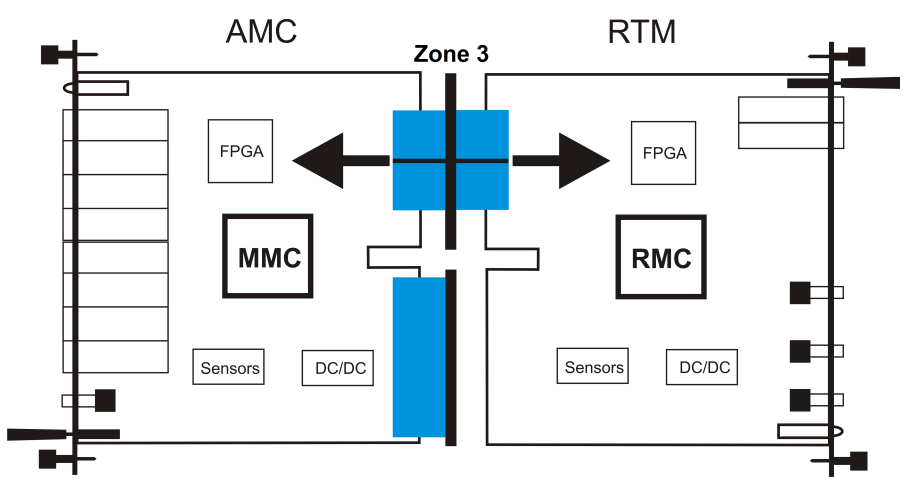

Fig. 2. An Advanced Mezzanine Card with connected Rear Transition Module

required for the RTM card to support monitoring and hardware management.

\section{A. IPMI Management of an AMC Module}

A MMC controller is required for every AMC module. The MMC is responsible for communication with the Carrier Manager and monitoring of crucial AMC parameters, like: temperature, supply voltages and currents. It provides module hot-plug capability, payload management and controls the activation and deactivation process. The MMC provides electronic-keying mechanisms for backplane interfaces. In the 
MTCA.4 systems, it is also responsible for RTM management (FRU processing, power supply control, hot-plug procedure and sensors reading). The RTM card is available as a separate FRU with ID equal to 1 (an AMC card has FRU ID equal to 0). The MMC is usually built with a simple microcontroller [12] [14].

\section{B. IPMI Management of an RTM}

A similar management circuit is required also for the RTM card. The RMC is responsible for power and payload control and provides access to sensors available on the card. It could also provide the advanced functions like: E-keying for the Zone 3 interfaces or payload firmware upgrade. The RMC device is fully controlled by the MMC present on the AMC. Therefore, the RMC is usually a simple circuit based on an $\mathrm{I}^{2} \mathrm{C}$ expander (the version proposed by the MTCA.4 spec.) or an 8-bit microcontroller.

\section{Available solutions}

Various implementations of MMC are available from commercial companies and research institutions [15]-[19]. Most of them are suitable for MTCA systems. However, none of these fully support RTM or provide extended functionalities, like: the E-keying for the Zone 3 or firmware upgrade for the MMC processor or an AMC payload (FPGA or CPU).

\section{MMC V1.00 - STANDARDIZED SOLUTION FOR MANAGEMENT CONTROLLER}

The solution presented in this paper, called MMC v1.00, is a unified template for Management Controllers for AMC and RTM boards. The goal of this project is to develop a readyto-use solution for MMC and RMC including both hardware (schematics, PCB layouts) and firmware designs. The project is addressed to designers of modules for MTCA-based systems not familiar with management requirements. They may use the available components directly in their projects to greatly shorten the time required for hardware design, software development and debugging. The other goal is the unification of various existing implementations of management controllers. The project provides a standardised solution for MMC and RMC controllers that covers most of the functionalities required by MTCA.4-compliant modules. Therefore, the MMC v1.00 product may be used without any changes in a typical, simple module or may be a good starting point for designing more dedicated solutions for custom-specific applications.

The project includes two versions (basic and advanced) for both MMC and RMC. The basic versions are dedicated for simple AMC and RTM modules and provide limited functionalities, e.g. number of sensors and channels for power supply control and monitoring. Advanced versions of both controllers are dedicated for complex and more demanding modules.

The block diagram of the AMC and RTM management controllers is presented in Figure 3. The MMC is based on an Atmel ATxmega microcontroller. The microcontroller is connected directly to the supervising system of the MTCA chassis (via the IPMI bus) and implements all the required MMC controller features. The microcontroller reports the current status of the board, allows to control the power supply and to manage the module operational states. Moreover, it monitors the operational parameters of the board i.e. temperature, voltage, current using set of sensors connected to a dedicated peripheral $\mathrm{I}^{2} \mathrm{C}$ bus. It is also responsible for Field Programmable Gate Array (FPGA) management and monitoring. The microcontroller provides also functions for handling the RTM module. It manages the power supply of the RTM using a dedicated power supply controller and controls its operational state.

Within the project, two evaluation boards (eval-kits), AMC and RTM, have been designed and manufactured (see Figure 7). The boards are a valuable reference for users of the MMC v1.00. They implement the proposed hardware solution of the MMC and RMC and may be useful to familiarize with operation principles of the management controllers. They are also a good test platform allowing development and verification of additional, customized functions of the MMCs.

\section{A. Module Management Controller}

The design of the AMC evaluation board has to meet a number of requirements, coming not only from a set of xTCA related specifications but also from recommendations provided by DESY and TUL. Compliance with AMC, MTCA and MTCA.4 standards requires the implementation to provide the following functions:

- support for the IPMB-L bus $\left(\mathrm{I}^{2} \mathrm{C}\right.$ based),

- handling of IPMI user interface (AMC handle and LEDs),

- presence indication and control over MCU reset,

- power supply management, including the RTM module,

- support for SDR and FRU records.

Further requirements are coming from the long term experience from developing xTCA hardware. These are related mainly to testing new concepts and maintaining compatibility between modules. The MMC eval-kit should:

- offer a number of temperature, voltage and current sensors,

- allow using complex JTAG chain configurations,

- support PMBus interface, for communication with modern power supplies,

- enable on-demand FPGA reconfiguration and different memories,

- be equipped with a globally unique digital identifier,

- provide a UART serial interface to ease debugging,

- provide a digitally keyed $\mathrm{I}^{2} \mathrm{C}$ bus to the RTM.

The MMC functions will be discussed in more detail in the following sections. A quick overview of provided functions is shown in Figure 4.

1) Module Management: Every AMC module has to provide a set of obligatory management functionalities. From the operator point of view, the AMC management may seem limited to reading the handle status and driving several LEDs. These easily observable features are however supported by nontrivial hardware and software solutions. 


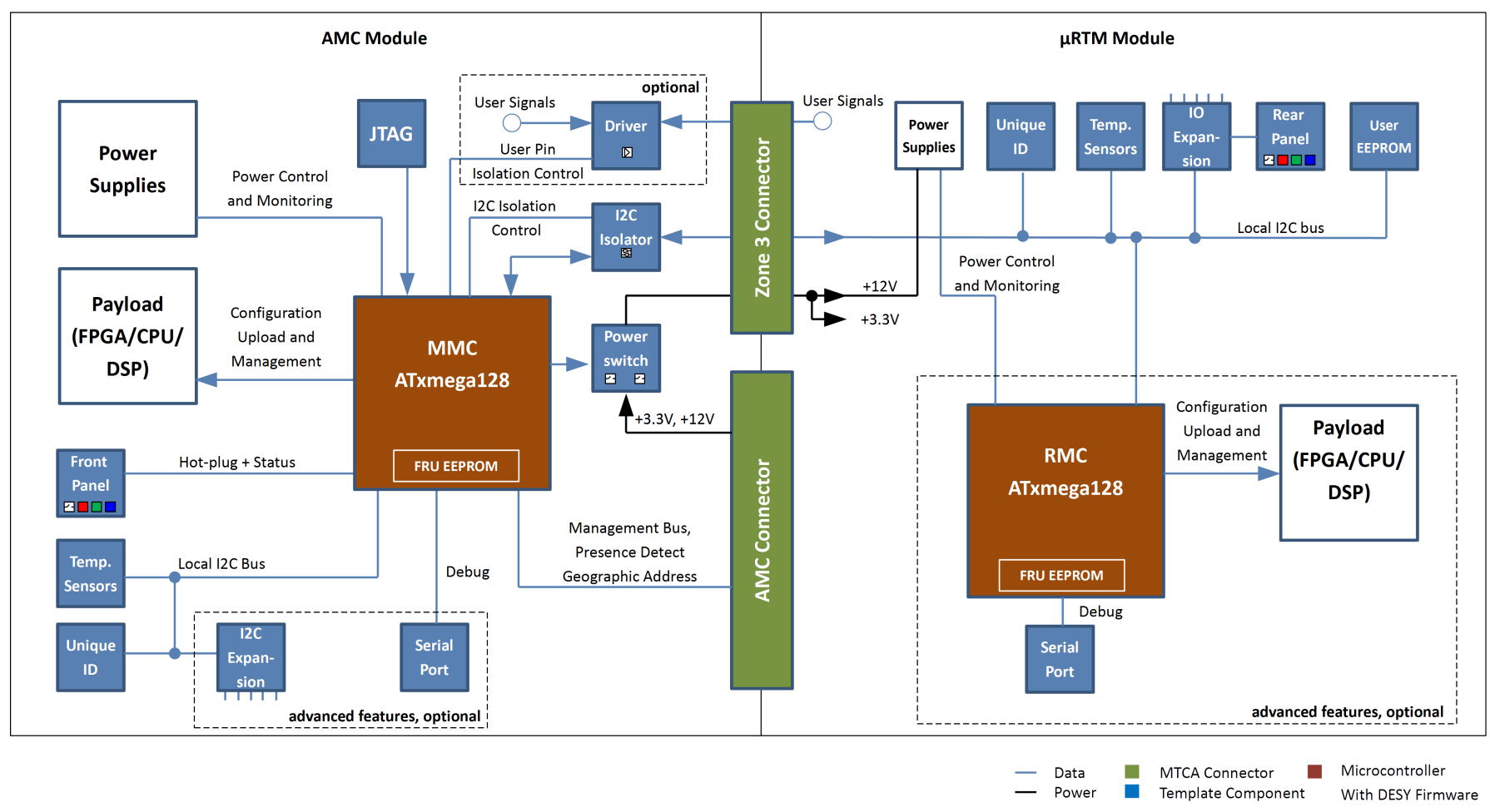

Fig. 3. A block diagram of the AMC and RTM management

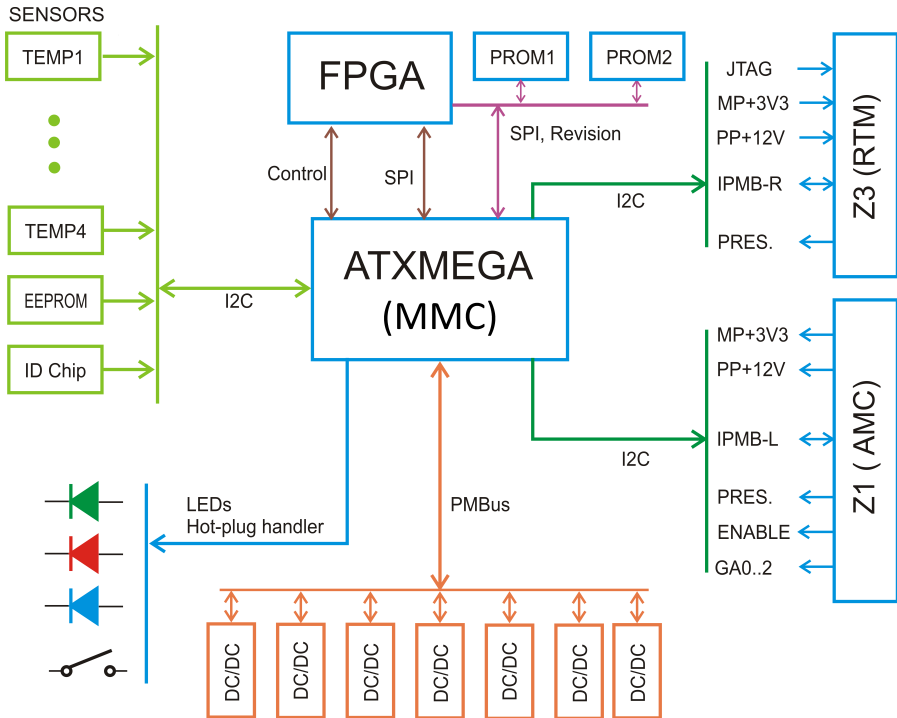

Fig. 4. A block diagram of the MMC management

When the AMC module is inserted into the crate, its activation process begins. The module is provided with $3.3 \mathrm{~V}$ power for management circuitry and the MMC is asked to identify the board. During this process the MMC returns the IPMI FRU record which contains information on board identification, available links and power consumption. Then the module waits for the fastening mechanism to lock. During this stage the blue LED shall be constantly lit. The activation process continues only after the handle is closed. Then, the blue LED starts blinking and the board may receive payload power. After the payload is ready for operation, the blue LED turns off and the operational status is further indicated by red and green LEDs.

The activation procedure, accompanied by an analogous deactivation process, ensures that the board may be safely inserted or removed even during system operation. The events generated by the hot-plug handle are used to open and close the communication links gracefully so modules may be exchanged without interrupting the work of other components.

2) System Diagnostics: The MMC is responsible for continuous monitoring of board state. Therefore, the MMC measures the key operational parameters like temperature, power supply voltages and current by means of dedicated sensors. The presented MMC solution provides four sensors to measure temperature of the board in critical places and one dedicated to monitor FPGA internal temperature. It can also measure up to eight voltages using $\mathrm{A} / \mathrm{D}$ converters built into the microcontroller. Moreover, it allows monitoring of power supply circuits e.g. DC/DC converters by reading the current states of 'Power Good' signals and/or using the PMBus protocol. The architecture is scalable, hence the monitoring circuits can be easily adjusted for different configurations of payload. The MMC is also supervising the power supply of the RTM module. It continuously monitors both $3.3 \mathrm{~V}$ and $12 \mathrm{~V}$ voltages of the RTM and its power consumption.

According to the IPMI standard, information about the onboard sensors shall be provided to the Carrier and System Managers that are responsible for monitoring of the system, including all of its components. For this reason, the MMC has to implement dedicated structures, SDRs, that contain information about sensors types, units, thresholds and equations for 
data conversion. The presented solution provides a description of all aforementioned sensors in the form of the SDR structures and supports IPMI commands for getting information from the SDR repository and reading the current sensors values.

3) Power Supply Management: The MMC is responsible for controlling the operation of the payload power supplies. First it negotiates the power consumption with the $\mathrm{MCH}$. If the negotiation process completes successfully the board is provided with $12 \mathrm{~V}$ and can start activating the payload. Most FPGA circuits require enabling the power supplies in a predefined manner and the sequencing is usually done by the MMC microcontroller. This fact explains why the MMC circuit is usually equipped with several signals enabling various power domains.

The designed eval-kit allows the developer to explore all the mentioned possibilities of controlling the power supplies.

4) Payload Management: Usually the AMC module hosts an FPGA device, dedicated processor or high speed communication equipment. These devices may also require state management. In the simplest case it may be just a reset signal issued after powering the device on. In the case of the FPGA circuit it is common to control its booting process by driving the Reconfiguration Request line and monitoring the Configuration Done signal. Using this approach, the FPGA or other processing devices, can be rebooted upon request. Moreover, the described scheme may be used for easy upgrade of the payload device firmware.

The developed eval-kit contains a slot for a payload circuit (e.g. FPGA) and two on-board non-volatile memories for booting the circuit. The board is equipped with PCIe and GbE connectivity for development of fast upgrade procedures. The SPI memories can be also accessed by the MMC, allowing implementation of the rescue procedure of programming memories over IPMI.

5) RTM Controller: The RTM module, defined by the MTCA.4, is another sub-system that requires state management. The operations of the hot-swap handle and LEDs are almost identical. Despite that the RTM board can contain a dedicated management microcontroller and control over RTM activation process is governed by the MMC. The circuit is responsible for powering the management power bus for the RTM, enforcing current limitation, checking its compatibility and hot-swap handle state and activating the module when appropriate.

The procedure requires continuous monitoring of module presence, control of the RTM power supply and communication with the RTM management circuity for controlling LEDs. The operation of the module can also be monitored in terms of power supply voltages and consumed current. The AMC evaluation board allows implementation of all the mentioned features. Moreover, it also enables automatic inclusion of the RTM JTAG chain into the AMC board JTAG, by means of a general purpose CPLD circuit.

6) System Debug: The MMC firmware can be debugged using several interfaces. Simple textual messages can be sent using a virtual serial port, available through USB connection. The actual firmware debugging can be performed using JTAG or PDI interfaces, both available at the same Atmel standard- ized connector. Observation of bus signals is eased by placing a monitoring sockets in every $\mathrm{I}^{2} \mathrm{C}$ bus and on some other signals of high importance.

The payload can be accessed for debugging using a 28-pin Xilinx compatible JTAG port, available on the front panel. Some of the most common issues can be visually diagnosed by observing LED indicators on the front panel.

\section{B. RTM Management Controller}

The RTM Management Controller is a passive device from the MTCA management point of view. It does not communicate directly with the supervising system, but provides only information about the RTM for MMC's simple monitoring [11]. Thus, the basic RTM management controller includes:

- I/O Expander connected to RTM control signals (e.g. MTCA-specific front panel LEDs and Hot Swap Handle),

- temperature sensor,

- non-volatile EEPROM for FRU records storage and unique ID EEPROM,

- $\mathrm{I}^{2} \mathrm{C}$ communication with the AMC,

- optional sensors: e.g. A/D converters for on-board voltage readings.

The block diagram of the basic RMC is presented in Figure 5.

The basic version of the RMC proposed in the MTCA.4 specification is fully sufficient for simple RTM boards like signal conditioners or adapters. However, in case of more complex boards like high-performance A/D or D/A converters or data processing modules based on FPGA devices, more advanced management and monitoring is required. Such boards require various sensors for monitoring of temperature or power supply voltages. Moreover, the control of power supply units (e.g. DC/DC converters) and supervising of payload devices (e.g. ADC, DAC, FPGA) is required. All these reasons have led the authors to design the advanced version of the RTM management controller ensuring extended functionality. The advanced solution of the RMC is based on the ATxmega microcontroller that acts as a bridge between the MMC and the sensors/actuators present in the RTM. The microcontroller communicates with all other implementation-specific sub-systems and provides a set of internal registers for its configuration and readout. The advanced solution also includes all components of the basic version i.e. $\mathrm{I}^{2} \mathrm{C} \mathrm{I} / \mathrm{O}$ expander, temperature sensors etc. Therefore, it is fully compatible with the MTCA.4 specification and the microcontroller is treated as an additional sensor that may be used by compatible AMC modules. The block diagram of the advanced RMC is presented in Figure 6. The additional functions that may be implemented using the microcontroller are:

- voltage monitoring using built-in ADCs,

- control of power supply units (e.g. using PMBus protocol),

- supervising of payload devices (e.g. FPGA) - initialization, rebooting, reset, etc.,

- providing information about the module (e.g. serial number, FPGA firmware version, etc.). 


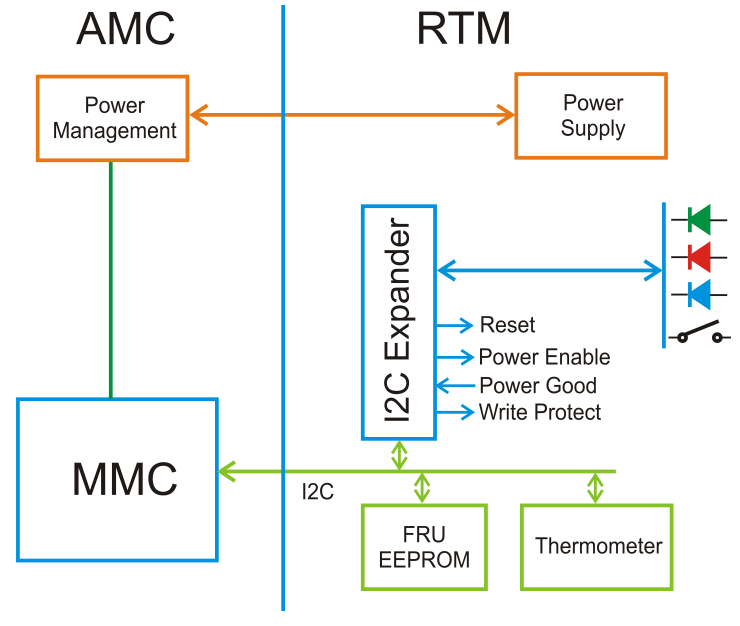

Fig. 5. A block diagram of the basic RTM management attached to the MMC

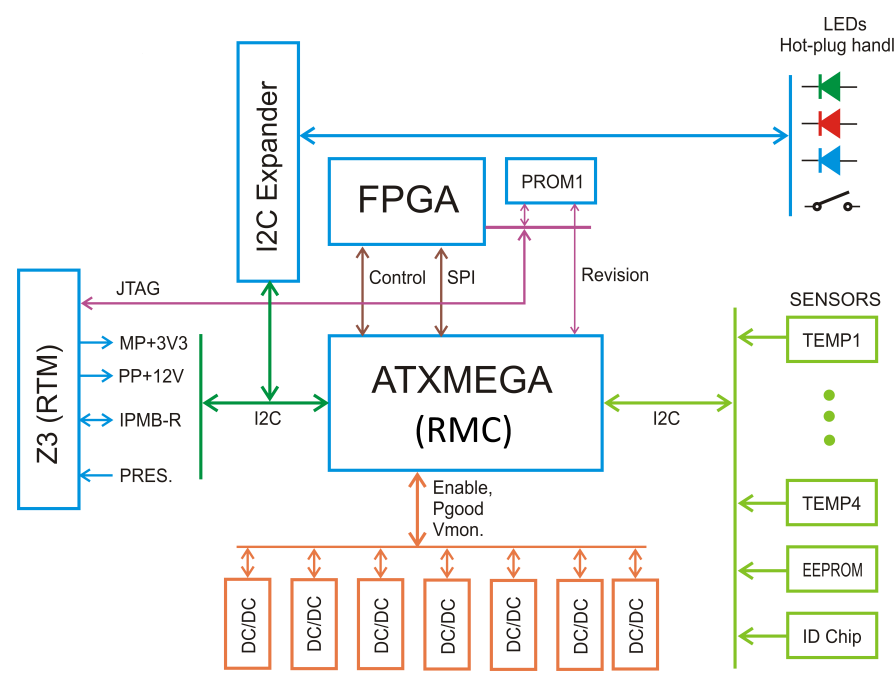

Fig. 6. A block diagram of the advanced RTM management

1) Module Management: Components of the RMC required for module management are common for both basic and advanced versions. This implementation is based on the circuit proposed by the MTCA.4 standard [11]. Two main elements required for RTM management are the I/O expander and EEPROM memory. They are connected to the $\mathrm{I}^{2} \mathrm{C}$ bus managed by the AMC board. Both devices have standardized $\mathrm{I}^{2} \mathrm{C}$ addresses; therefore they can easily be found by the MMC. The memory is dedicated for storing FRU identification information. The information is read by the MMC during RTM module initialization. The $\mathrm{I} / \mathrm{O}$ expander with the $\mathrm{I}^{2} \mathrm{C}$ interface is used to control and monitor standard RTM management signals. The expander is connected to three MTCA-specific LEDs on the front panel, Hot Swap Handle, reset signal and common signals for supervising of all power supply units.

2) System Diagnostics: The RTM operational parameters are monitored by the MMC using sensors connected to the $\mathrm{I}^{2} \mathrm{C}$ bus dedicated for communication with the RTM. The basic version of the RMC provides only temperature sensors. In the advanced version of the RMC the ATxmega microcontroller has been used as an additional slave device connected to the RTM $\mathrm{I}^{2} \mathrm{C}$ bus. It extends the diagnostics and control functionality. It allows measuring of eight on-board voltages (using built-in A/D converters) and monitoring of individual status signals from power supply units e.g. DC/DC converters.

3) Payload Management: The RMC also allows management of payload devices placed on the RTM board. The management covers both power supply supervising and control of device operation. In the basic version only two signals ('Reset' and 'Power Enable') connected to the I/O expander may be controlled. The 'Reset' signal may be used to reset all payload devices (e.g. FPGA) and the 'Power Enable' signal is dedicated to control of the power supply i.e. enabling/disabling of DC/DC converters. The advanced version RMC supports up to 6 individual 'Power Enable' signals that may be controlled with the microcontroller. In this version also the supervising of the FPGA device is extended. The microcontroller allows controlling and monitoring of FPGA configuration signals (i.e. 'DONE', 'INIT_B', 'PROG_B' and 'RESET'). All these signals may be used by the MMC for FPGA restarting or firmware reloading.

4) Zone 3 Connectivity Management: The AMC and RTM boards are connected with two 30-pair ADF connectors in Zone 3. Although the positions of these connectors are specified by the MTCA.4 standard, the signal-to-pin mapping is defined only for IPMI, JTAG and Power connections. To avoid compatibility problems between various boards, Z3 Connector pin assignment recommendation have been proposed [20]. These recommendations define unified classes for digital and analogue boards. The classes describe the layouts of Zone 3 connectors taking into account signal levels and communication standards. The boards from different classes may be incompatible; therefore one of the tasks of the MMC and RMC is to provide E-Keying. The MMC is responsible for reading data from the EEPROM memory on the RTM and checking if this board is compatible with the AMC. The verification is done on the basis of a dedicated compatibility record providing precise description of the Zone 3 interface.

\section{Firmware Upgrade}

The MMC v1.00 allows reprogramming of the ATxmega microcontroller that is the heart of the proposed implementation. The firmware upgrade has been implemented according to the Hardware Platform Management IPM Controller Firmware Upgrade Specification (HPM.1) [21]. The specification defines a firmware upgrade procedure dedicated for all IPMIcompliant controllers and may be applied to management controllers used in all xTCA standards. The described MMC implementation supports additional commands needed to perform firmware upgrade according to HPM.1 specification.

The MMC can also participate in reprogramming of FPGA devices placed on the AMC and RTM modules, as mentioned earlier. The detailed procedure of firmware upgrade can be found in [22].

\section{MMC V1.00 - PRACTICAL IMPLEMENTATION}

\section{A. Eval-kits for Rapid Development of IPMI Controllers}

The assembled AMC-Eval Kit module with attached RTMEval Kit is presented in Figure 7. The boards were first tested 
separately, powered by a laboratory power supply. After the basic functionality was achieved, operation of the complete set was verified in the MTCA.4 compliant shelf, using the MMC code developed by TUL-DMCS. The further tests were performed with the MTCA.4 12-slot chassis from Schroff and the MCH from NAT [23].

1) Module Management Controller: Tests of the AMCEval Kit module starts with powering the management power domain and verifying microcontroller operation. The JTAG port and UART are tested first, followed by tests of mandatory AMC elements and front panel LEDs. When this part is completed the Payload Power is provided and the testing procedure focuses on verification of DC/DC converters and linear regulators operation.

2) RTM Management Controller: Tests of the RTM-Eval Kit are very similar to those of the AMC module. These start with verification of the microcontroller operation, and then focus on the power supplies. When this is done, the operation of the FPGA module is verified. Finally correctness of the sensors readouts is verified in the MTCA. 4 shelf.

\section{B. Example Applications}

The photo of DAMC-TCK7 with attached DRTM-VM02LF module is presented in Figure 8. This set of boards will be used to control the accelerator part of the XFEL laser. The boards were developed in collaboration between DESY, TUL and WUT (Warsaw University of Technology).

1) DAMC-TCK7 - Data Processing Module: The AMCbased Controller (DAMC-TCK7) board is a general purpose high-performance low-latency data processing unit designed according to the MTCA.4 specification [24]. The module provides processing power, data memory, communication links and reference clock signals. The FPGA device available on the DAMC-TCK7 board delivers computing power for a lowlatency digital signal processing. The FPGA supports a number of Low Latency Links (LLL) available on the front panel, at the backplane and at the Rear Transition Module (RTM) Zone 3 connector, working with a few Gigabits throughput. Since the control algorithms can be improved in the future, the device supports in-system firmware upgrade, using IPMI and the fast serial link. The module is the updated version of the $\mu \mathrm{TC}$ processing module [14].

2) DRTM-VM02LF - Vector Modulator Module: The DRTM-VM02LF is a two-channel low-frequency vector mod-

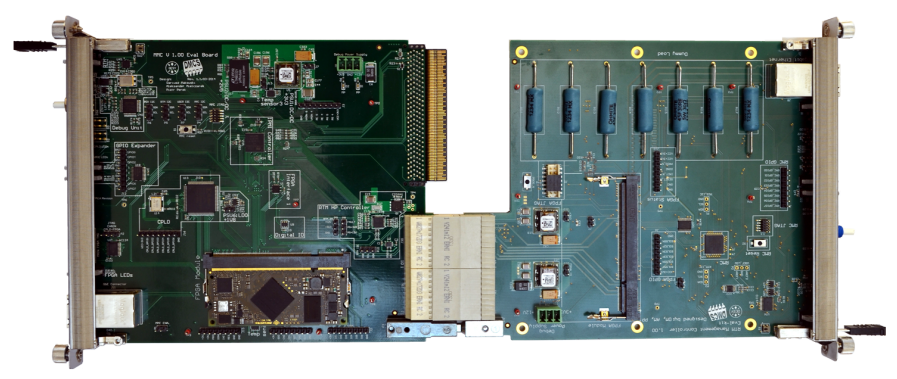

Fig. 7. The photo of the AMC and RTM eval-kits

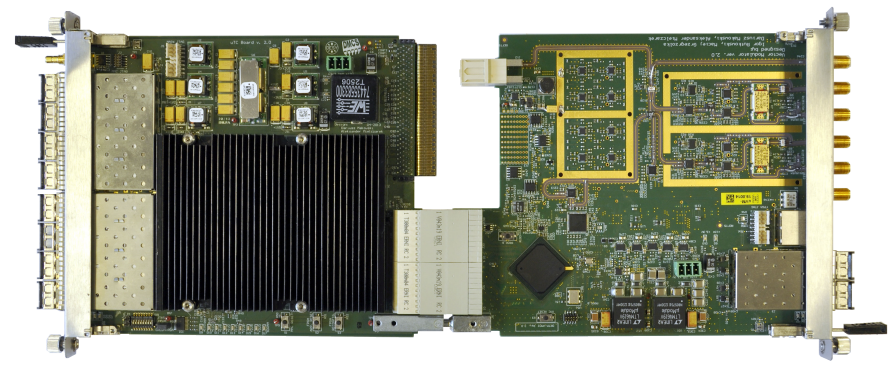

Fig. 8. A photograph of DAMC-TKC7 module with attached the DRTMVM02LF module

ulator card dedicated for RF control systems [25]. The DRTMVM02LF module is designed as an RTM. The DRTMVM02LF module incorporates digital, analogue and diagnostic subsystems. The digital part is based on the Xilinx Spartan 6 family FPGA, with several fast gigabit-link connections to a signal processing/control module. The analogue part includes quadrature amplitude modulators and clock management circuity running at $1.3 \mathrm{GHz}$.

\section{MMC Firmware}

The management firmware for both, DAMC-TCK7 and DRTM-VM02LF boards, has been developed based on the solution described in [7]. The firmware implements all functionalities necessary for operation in a MTCA.4 systems. It supports communication based on the IPMI protocol and ensures monitoring of on-board voltages, temperatures and power consumption, management of FPGA and firmware upgrade. It also provides handling of RTM boards and Ekeying mechanism for Zone 3 interconnections as well as a UART for debugging and local monitoring.

\section{Conclusions and Plans for Future}

The MMC v1.00 solution is the first attempt to provide unified IPMI templates for AMC and RTM cards. Schematics diagrams, hardware and initial implementation of firmware were developed. The implementations were evaluated and tested with designed Eval Kit boards that allow fast development of IPMI management for MTCA.4 modules. The MMC v1.00 templates were applied to the first modules of the LLRF control system of the European XFEL accelerator: lowlatency data processing module (DAMC-TCK7) and Vector Modulator (DRTM-VMLF).

The current solution covers all basic functionality required by the MTCA.4 specification. It also provides new functions useful for development and application of hardware used in High Energy Physics experiments. However, the firmware for the MMC is still under development and new, more advanced functions are now being added. In the next steps, the authors are planing to prepare a framework for firmware developers. The framework will provide generic functionality, common for all MTCA.4 modules. It will be freely modifiable, allowing tailoring the proposed solution to the requirements of the specific module. 


\section{ACKNOWLEDGMENT}

This project is supported by Grant No. HVF-0016 "MTCA.4 for Industry" awarded by the Helmholtz Validation Fund.

\section{REFERENCES}

[1] PICMG, "Micro Telecommunications Computing Architecture," July 2006.

[2] D. Makowski, W. Koprek, T. Jezynski, A. Piotrowski, G. Jablonski, W. Jalmuzna, K. Czuba, P. Predki, S. Simrock, and A. Napieralski, "Prototype Real-Time ATCA-based LLRF Control System," Nuclear Science, IEEE Transactions on, vol. 58, no. 4, pp. 1553-1561, aug. 2011.

[3] P. Predki, D. Makowski, and A. Napieralski, "Intelligent PlatformManagement Controller for Low-Level RF Control System ATCA Carrier Board," Nuclear Science, IEEE Transactions on, vol. 58, no. 4, pp. 1538-1543, aug. 2011.

[4] R. Larsen, "PICMG xTCA standards extensions for Physics: New developments and future plans," in Real Time Conference (RT), 2010 17th IEEE-NPSS, May 2010, pp. 1-7.

[5] J. Branlard, G. Ayvazyan, V. Ayvazyan, M. Grecki, M. Hoffmann, T. Jezynski, F. Ludwig, U. Mavric, S. Pfeiffer, H. Schlarb, C. Schmidt, H. Weddig, B. Yang, P. Barmuta, S. Habib, L. Butkowski, K. Czuba, M. Grzegrzolka, E. Janas, J. Piekarski, I. Rutkowski, D. Sikora, L. Zembala, M. Zukocinski, W. Cichalewski, W. Jalmuzna, D. Makowski, A. Mielczarek, A. Napieralski, P. Perek, A. Piotrowski, T. Pozniak, K. Przygoda, G. Boltruczyk, S. Korolczuk, M. Kudla, J. Szewinski, K. Oliwa, and W. Wierba, "MTCA.4 LLRF system for the European XFEL," in Mixed Design of Integrated Circuits and Systems (MIXDES), 2013 Proceedings of the 20th International Conference, June 2013, pp. $109-112$.

[6] PICMG, "AdvancedMC Mezzanine Module specification PICMG AMC.0," Nov. 2006.

[7] P. Perek, A. Mielczarek, P. Predki, D. Makowski, and A. Napieralski, "Module Management Controller for MicroTCA-based Controller Board," in Mixed Design of Integrated Circuits and Systems (MIXDES), 2011 Proceedings of the 18th International Conference, June 2011, pp. 159-164.

[8] D. Makowski, "The Importance of Intelligent Platform Management Controller for LLRF system," in Review of LLRF system based on ATCA standard, 8-9 Nov 2007.

[9] Intel, HP, NEC, and DELL. (2013, October) IPMI v2.0 rev. 1.1 specification. [Online]. Available: http://www.intel.com/design/servers/ipmi/spec.htm/

[10] PICMG, AdvancedTCA Base Specification, Jan. 2003, PICMG 3.0.

[11] PICMG, "MicroTCA Enhancements for Rear I/O and Precision Timing," August 2011.
[12] D. Makowski, A. Piotrowski, and A. Napieralski, "Universal communication module based on AMC standard," in 15th International Conference on Design of Integrated Circuits and System, 2008. MIXDES 2008. Proceedings of the International Conference, Jun. 2008, pp. 139143.

[13] Struck, "SIS8300 uTCA for Physics Digitizer - User Manual," Struck innovative systems, Harksheider Str. 102A, Hamburg, Germany, Tech. Rep. 1.02, November 2010.

[14] A. Mielczarek, D. Makowski, G. Jablonski, A. Napieralski, P. Perek, P. Predki, T. Jezynski, F. Ludwig, and H. Schlarb, "uTCA-based Controller," in Mixed Design of Integrated Circuits and Systems (MIXDES), 2011 Proceedings of the 18th International Conference, June 2011, pp. 165-170.

[15] P. Perek, A. Mielczarek, P. Predki, D. Makowski, and A. Napieralski, "Module management controller for MicroTCA-based controller board," in Mixed Design of Integrated Circuits and Systems (MIXDES), 2011 Proceedings of the 18th International Conference, June 2011, pp. 159164.

[16] Pigeon Point. (2014) AdvancedMC Module Management Controller. [Online]. Available: http://www.pigeonpoint.com/products_picmg.html

[17] Kontron. (2013, Nov.) AMC-MMC Developer Kit. [Online]. Available: http://www.kontron.com/products/boards-andmezzanines/advancedmc/i-o/amc-mmc-developer-kit.html

[18] P. Kammerling, M. Drochner, H. Kleines, S. van Waasen, M. Ramm, and A. Ackens, "Development of an AMC Module Management Controller," in Real Time Conference (RT), 2012 18th IEEE-NPSS, June 2012, pp. $1-3$.

[19] J. Cachemiche. (2011, March) MMC Mezzanine Board Specifications. [Online]. Available: https://espace.cern.ch

[20] F. Ludwig, D. Makowski, and H. Schlarb, "Zone 3 Classification for MicroTCA.4," in Real Time Conference (RT), 2014 19th IEEE-NPSS, 2014.

[21] PICMG, "Hardware Platform Management," June 2007.

[22] D. Makowski, G. Jablonski, P. Perek, A. Mielczarek, P. Predki, H. Schlarb, and A. Napieralski, "Firmware Upgrade in xTCA Systems," Nuclear Science, IEEE Transactions on, vol. 60, no. 5, pp. 3639-3646, Oct 2013.

[23] N.A.T., "NAT-MCH Users Manual," March 2010.

[24] D. Makowski, A. Mielczarek, P. Perek, J. Branlard, M. Fenner, H. Schlarb, L. Butkowski, B. Yang, and A. Napieralski, "High-Speed data processing module for LLRF," in Real Time Conference (RT), 2014 19th IEEE-NPSS, 2014.

[25] I. Rutkowski, K. Czuba, D. Makowski, A. Mielczarek, H. Schlarb, and F. Ludwig, "Vector Modulator Card for MTCA-Based LLRF Control System for Linear Accelerators," Nuclear Science, IEEE Transactions on, vol. 60, no. 5, pp. 3609-3614, Oct 2013. 